\title{
Ligustrazinyl amides: a novel class of ligustrazine-phenolic acid derivatives with neuroprotective effects
}

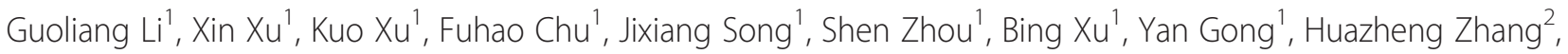
Yuzhong Zhang ${ }^{2}$, Penglong Wang ${ }^{1 *}$ and Haimin Lei ${ }^{1 *}$

\begin{abstract}
Background: Ligustrazine has potent effects of thrombolysis, neuroprotection and vascular protection, which were important for effectively protecting the nervous system. Previous study in our laboratory reported that ligustrazine-benzoic acid derivatives have been shown to exhibit beneficial effect against $\mathrm{CoCl}_{2}$-induced neurotoxicity in differentiated PC12 cells. To further improve ligustrazine's neuroprotection, we integrated the ligustrazine and phenolic acid fragments into one molecule via an amide bond based on structural combination.

Results: In this study, 12 novel ligustrazine-phenolic acid derivatives were synthesized and nine others were prepared by improved methods. Furthermore, these compounds were evaluated for their protective effects against $\mathrm{CoCl}_{2}$-induced neurotoxicity in differentiated PC12 cells. The amides conjunctional derivatives exhibited promising neuroprotective activities in comparison with ligustrazine. In addition, the most active congener (E)-3-(2,3,4-trimethoxyphenyl)-N-((3,5,6-trimethylpyrazin-2-yl)methyl)acrylamide (L10, EC $50=25 \mu \mathrm{M})$, which is 2 times higher than that of ligustrazine, may be a potential candidate for intervention in neurological diseases. Structure-activity relationship was discussed briefly.

Conclusions: Results of series of ligustrazinyl amides enrich the study of ligustrazine derivatives with neuroprotective effects. Our completed work supports that the attempt to apply structure combination to discover more efficient neuroprotection lead compounds is viable.
\end{abstract}

Keywords: Ligustrazine derivative, Phenolic acid, Neuroprotective effect, PC12 cell

\section{Background}

Neurological disorders, such as Stroke, Alzheimer's disease (AD) and Parkinson's disease (PD), threaten millions of patients with ever growing numbers in ageing societies [1-3]. To discover drugs with nerve functional recovery as a treatment or prevention of neurological disorder is of great significance [4]. To date, despite the remarkable progress achieved in theory, effective approaches to recover damaged nerve are not yet to be found $[1,5]$. Therefore, to discover more effective drugs for the treatment in injured nerve cell remains an important area of drug discovery [6,7].

\footnotetext{
* Correspondence: wpl581@126.com; hm_lei@126.com

'School of Chinese Pharmacy, Beijing University of Chinese Medicine, No.6 Wangjing Middle Ring South Road, Beijing, Chaoyang District, China Full list of author information is available at the end of the article
}

Nowadays, many new drugs have been generated from natural products [8-11]. Ligustrazine (2,3,5,6tetramethylpyrazine, TMP), derived from the traditional Chinese medicine Rhizoma Chuanxiong (Ligusticum chuanxiong Hort.) which was widely used to treat Stroke and cerebrovascular disease (CVD) in China $[12,13]$. Recent studies have indicated that ligustrazine has potent effects of thrombolysis, neuroprotection and vascular protection, which were important for effectively protecting the nervous system [14-21]. In addition, many phenolic acid ingredients, such as caffeic acid, protocatechuic acid, salicylic acid, ferulic acid, vanillic acid, etc., also showed interesting neuroprotective activities [21-24].

To further improve ligustrazine's neuroprotective effect, inspired by the potent neuroprotective effects of 
ligustrazine-benzoic acid derivatives, we integrated the ligustrazine and phenolic acid fragments into one molecule based on structural combination [17,21,25]; a series of novel ligustrazine-benzoic analogues was constructed via an amide bond rather than an ester bond in our previous research on ligustrazine-benzoic acid derivatives. A recent study has reported that part of ligustrazinyl amides congener structures exhibited good proliferative activities on human umbilical vascular endothelial cells (HUVECs) [25]. Their protective effects against neurotoxicity were evaluated in differentiated PC12 cells. Structure-activity relationship was discussed briefly.

\section{Results and discussion}

\section{Chemistry}

All the target compounds were synthesized via the routes outlined in Scheme 1, Scheme 2 and Scheme 3. The key intermediate (3,5,6-trimethylpyrazin-2-yl)methanamine (L) was prepared according to our previous study with minor improvements. Compound B (TMP-Br) was synthesized from anhydrous ligustrazine and $\mathrm{N}$-bromosuccinimide (NBS) in carbon tetrachloride via free radical reaction, the crude product was used directly in the next reaction without further purification. The mixture of TMP-Br and phthalimide potassium in $\mathrm{CH}_{3} \mathrm{CN}$ that was refluxing for $2 \mathrm{~h}$ gave compound $\mathbf{C}$. Intermediate $\mathbf{L}$ was obtained by reaction of $\mathbf{C}$ and $80 \%$ hydrazine hydrate in absolute ethanol refluxing for $5 \mathrm{~h}$.

The single-step coupling reaction between $\mathbf{L}$ and the cinnamic acids were performed using EDCI and $\left(\mathrm{CH}_{3} \mathrm{CH}_{2}\right)_{3} \mathrm{~N}$ in anhydrous $\mathrm{CH}_{2} \mathrm{Cl}_{2}$, to afford ligustrazine derivatives (L1-L10, as shown in Method 1 of Scheme 2). In Method 2 and 3, the carboxylic acids and HOBt were firstly transformed to active ester in the presence of EDCI, and then reacted with compound $\mathbf{L}$, obtaining the target compounds (L11-15, L17-21).

In Scheme 3, the starting compound $\mathbf{1 6}$ was first perbenzylated and then transformed to free carboxylic acid. The coupling reaction between $\mathbf{L}$ and the hydroxylperbenzylated benzoic acid 16b was performed using EDCI and HOBt in anhydrous $\mathrm{CH}_{2} \mathrm{Cl}_{2}$. A final deprotection step afforded the targeted compound L16. The chemical structures of all target compounds (Table 1) were confirmed by ${ }^{1} \mathrm{H}$-NMR, ${ }^{13} \mathrm{C}$-NMR and high resolution mass (HRMS).

\section{Biological activities}

\section{Protective effect on injured neuronal-like PC12 cells}

Setting ligustrazine as the positive control drug, all the synthesized compounds were tested for their protective effects on neuronal-like PC12 cells damaged by $\mathrm{CoCl}_{2}$. The proliferation rates (P\%) of injured $\mathrm{PC} 12$ cells were assessed by methyl thiazolyl tetrazolium (MTT) assay. The proliferation rates (\%) at different concentration and $50 \%$ effective concentrations $\left(\mathrm{EC}_{50}\right)$ for protecting damaged PC12 cells of the ligustrazine derivatives were outlined in Table 2.

From the obtained results, it was observed that ligustrazine and most of its derivatives presented protective effects on injured differentiated PC12 cells, and several ligustrazine derivatives exhibited competitive positive activities (with lower $\mathrm{EC}_{50}$ values) than $\mathrm{TMP}\left(\mathrm{EC}_{50}=\right.$ $65 \mu \mathrm{M})$. Among them, L10 and L11 displayed promising neuroprotective activities $\left(\mathrm{EC}_{50}=25,27 \mu \mathrm{M}\right.$, respectively), in which compound $\mathbf{L 1 0}$ presented 2 times higher potency than TMP.

Among L1-L10, compounds that introduced methyl and methoxy group on the phenyl ring performed better neuroprotective activities than L1 without any group substituted. It can be concluded that with the increase of the number of methoxy group at the phenyl moiety, the activities increase considerably $(\mathbf{L} 10>\mathbf{L 3}, \mathbf{L 6}, \mathbf{L 8}>\mathbf{L 4}$, L5, L7). However, this rule did not work in the case of L9 $\left(\mathrm{EC}_{50}=102 \mu \mathrm{M}\right)$ on the injured PC12 cells model. The structure-activity relationship analysis was consistent with the protective effects of ligustrazine-based stilbene derivatives on damaged ECV-304 cells [26]. These findings may provide a new framework for the design of new ligustrazine derivatives as neuroprotective drugs.

It should be noticed that ligustrazinyl amides L12L16 with phenolic hydroxyl substituted approximately followed a tendency in activity 4-OH, $3-\mathrm{OCH}_{3}>4-\mathrm{OH}>$ $2-\mathrm{OH}>3-\mathrm{OH}>3,4-\mathrm{OH}$. This was similar to our previous research that the ligustrazine-benzoic acid derivatives were synthesized via an ester bond [21]. Moreover, compounds L15 and L11 were derived from salicylic acid and acetylsalicylic acid, respectively. But L11 $\left(\mathrm{EC}_{50}=\right.$ $27 \mu \mathrm{M})$ displayed observable protective action, and it is

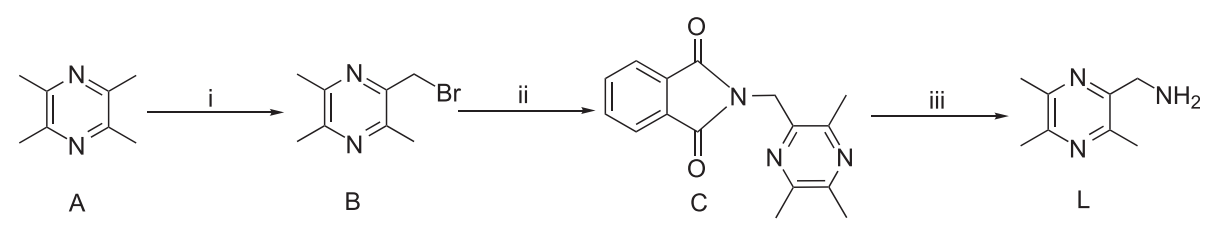

Scheme 1 Synthetic routes to ligustrazine intermediate L. Reagents and Conditions: (i) $\mathrm{CCl}_{4}, \mathrm{NBS}$, hv, reflux, 2 h, $65 \%$; (ii) $\mathrm{CH}_{3} \mathrm{CN}_{\text {, phthalimide }}$ potassium, reflux, $2 \mathrm{~h}, 64 \%$; (iii) $\mathrm{CH}_{3} \mathrm{CH}_{2} \mathrm{OH}, \mathrm{N}_{2} \mathrm{H}_{4} \cdot \mathrm{H}_{2} \mathrm{O}$, reflux, $5 \mathrm{~h}, 88 \%$. 


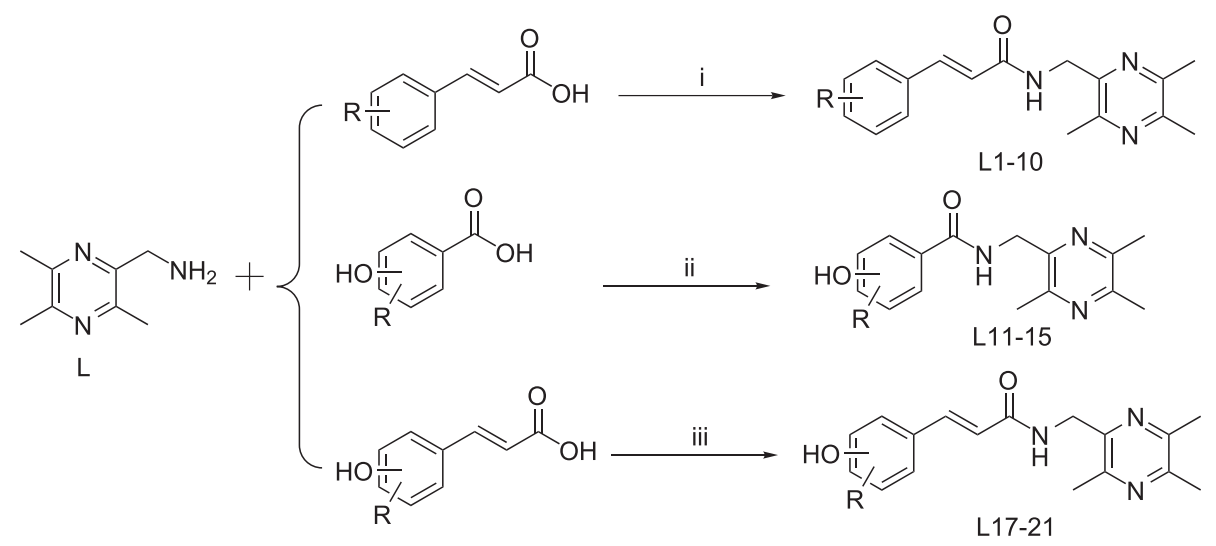

Scheme 2 Synthetic routes to ligustrazine derivatives L1-L15, L17-L21. Reagents and Conditions: (i) anhydrous $\mathrm{CH}_{2} \mathrm{Cl}_{2}, \mathrm{EDCl}\left(\mathrm{CH}_{3} \mathrm{CH}_{2}\right)_{3} \mathrm{~N}, \mathrm{r}$. t., 12 h; (ii) anhydrous DMF (L11 anhydrous $\mathrm{CH}_{2} \mathrm{Cl}_{2}$ ), EDCl/HOBt, r.t., 12 h; (iii) anhydrous DMF (L21 DMl), EDCl/HOBt, r.t., 12 h.

much better than $\mathbf{L 1 5}\left(\mathrm{EC}_{50}=92 \mu \mathrm{M}\right)$. This implied that acetyl group may be an effective group, led to improvement of the neuroprotective activities. In addition, the introduction of trans olefinic bond may contribute to enhance the neuroprotective activity, such as $\mathbf{L 2 0}>\mathbf{L 1 2}$, L17 > L13, L19 > L15 and L21 > L16.

Furthermore, previous studies proved that compounds L2, L4, L19 and L20 could stimulate the proliferation of cultured human umbilical vascular endothelial cells [25]; current study also exhibited that L2, L4, L19 and L20 exhibited neuroprotective activities. Based on the above evidences, we reason that the new synthetic ligustrazinylated derivatives possess multiple pharmacological activities such as neuroprotection and protection against vascular endothelial cell injury, suggesting that they may be more efficacious than either a neuroprotective agent or vascular protective drug alone.

\section{Effects of L10 on PC12 cells in morphology}

Observed under optical microscopy (OLMPUS, Japan), as shown in Figure 1-A, we found that undifferentiated
PC12 cells that maintained under normal conditions were small and proliferated to form clone-like cell clusters without neural characteristics. By exposure to NGF, normal differentiated PC12 cells showed fine dendritic networks similar to those nerve cells (Figure 1-B). In contrast, $\mathrm{CoCl}_{2}$-insulted differentiated PC12 cells developed mild cell body swelling and some cells shrunk the dendritic networks even lost neurites demonstrating round shape (Figure 1-C). Pretreatment with L10 alleviated morphological manifestations of cells damage compared to model cells (Figure 1-D).

\section{Conclusions}

In this work, 21 novel ligustrazine-phenolic acid derivatives were designed, synthesized and biologically evaluated for their protective effects against $\mathrm{CoCl}_{2}$-induced neurotoxicity in differentiated PC12 cells. The biological results have demonstrated that most of ligustrazine derivatives exhibited better neuroprotective activities in comparison with ligustrazine. In addition, the most active congener $\mathbf{L 1 0}\left(\mathrm{EC}_{50}=25 \mu \mathrm{M}\right)$, which is 2 times<smiles>CC(C)(C)c1ccc(OCc2ccccc2)c(OCc2ccccc2)c1OCc1ccccc1COC(=O)c1ccc(OCc2ccccc2)c(OCc2ccccc2)c1</smiles>

Scheme 3 Synthetic routes to ligustrazine derivatives L16. Reagents and Conditions: (i) DMF, benzyl bromide, $\mathrm{K}_{2} \mathrm{CO}_{3}, 85^{\circ} \mathrm{C}, 12 \mathrm{~h}$; (ii) $\mathrm{H}_{2} \mathrm{O} /$ $\mathrm{CH}_{3} \mathrm{CH}_{2} \mathrm{OH}, 10 \% \mathrm{KOH}, 70^{\circ} \mathrm{C}, 2$ h; (iii) TMP-NH $\mathrm{H}_{2}$ DMF, EDCl/HOBt, r.t., 12 h; (iv) $\mathrm{CH}_{3} \mathrm{OH}, \mathrm{Pd} / \mathrm{C}_{1} \mathrm{H}_{2}$, r.t., 12 h. 
Table 1 The structures of ligustrazine derivatives L1-21

\section{Structure}<smiles>Cc1nc(CNC(=O)/C=C/c2ccccc2)c(C)nc1[Al]</smiles><smiles>COc1ccc(/C=C/C(=O)NCc2nc(C)c(C)nc2C)cc1OC</smiles><smiles>COc1ccccc1/C=C/C(=O)NCc1nc(C)c(S)nc1C</smiles><smiles>COc1cccc(/C=C/C(=O)NCc2nc(C)c([Tl])nc2C)c1</smiles><smiles>COc1cc(/C=C/C(=O)NCc2nc(C)c(Br)nc2C)cc(OC)c1OC</smiles><smiles>CC(=O)Oc1ccccc1C(=O)NCc1nc(C)c([Al])nc1C</smiles><smiles>Cc1nc(C)c(CNC(=O)c2ccc(O)cc2)nc1C</smiles><smiles>Cc1nc(C(C)(C)C)c(C)nc1CNC(=O)c1ccccc1O</smiles>

\section{Yield}

$90.7 \%$<smiles>[Z10]c1nc(C)c(CNC(=O)/C=C/c2ccc(C)cc2)nc1C</smiles>

$86.3 \%$<smiles>[Z19]c1nc(C)c(CNC(=O)/C=C/c2ccc(OC)cc2)nc1C</smiles>

$90.1 \%$<smiles>[R]C(C)(C)c1nc(C)c(CNC(=O)/C=C/c2cccc(OC)c2OC)nc1C</smiles>

$91.3 \%$

$81.6 \%$<smiles>COc1ccc(OC)c(/C=C/C(=O)NCc2nc(C)c(C(Br)(Br)c3ccccc3)nc2C)c1</smiles><smiles>COc1ccc(/C=C/C(=O)NCc2nc(C)c([14C]#N)nc2C)c(OC)c1OC</smiles>

$68.9 \%$<smiles>[Z11]c1nc(C)c(CNC(=O)c2ccc(O)c(OC)c2)nc1C</smiles>

$76.9 \%$<smiles>Cc1nc(C)c(CNC(=O)c2cccc(O)c2)nc1C</smiles>

$78.4 \%$<smiles>Cc1nc(C(C)(C)C)c(C)nc1CNC(=O)c1ccc(O)c(O)c1</smiles>

Yield

$83.5 \%$

$85.8 \%$

$83.6 \%$

$88.5 \%$

$85.2 \%$

$81.8 \%$

$84.3 \%$ 
<smiles>CCCc1nc(C)c(CNC(=O)/C=C/c2ccc(O)cc2)nc1C</smiles>

$71.3 \%$<smiles>Cc1nc(CNC(=O)/C=C/c2cccc(O)c2)c(C)nc1[13CH3]</smiles><smiles>Cc1nc(CNC(=O)/C=C/c2ccccc2O)c(C)nc1[14CH3]</smiles><smiles>NC(=O)/C=C/c1ccc(O)c(O)c1</smiles><smiles>CCCc1nc(C)c(CN)nc1C</smiles>

$77.2 \%$<smiles>COc1cc(/C=C/C(=O)NCc2nc(C)c([18O])nc2C)ccc1O</smiles>

$74.8 \%$

$80.1 \%$
Table $2 \mathrm{EC}_{50}$ of the ligustrazine derivatives for protecting damaged PC12 cells

\begin{tabular}{|c|c|c|c|c|c|c|}
\hline \multirow[t]{2}{*}{ Compound } & \multicolumn{5}{|c|}{ Proliferation rate (\%) } & \multirow{2}{*}{$\begin{array}{l}\mathrm{EC}_{50} \\
(\mu \mathrm{M})\end{array}$} \\
\hline & $60 \mu \mathrm{M}$ & $30 \mu \mathrm{M}$ & $15 \mu \mathrm{M}$ & $7.5 \mu \mathrm{M}$ & $3.75 \mu \mathrm{M}$ & \\
\hline L1 & -20.01 & -14.71 & 5.47 & -8.63 & -15.83 & 150 \\
\hline L2 & 18.94 & 18.52 & 12.48 & 2.80 & -10.53 & 74 \\
\hline L3 & 38.69 & 14.19 & 12.04 & -0.57 & -7.52 & 64 \\
\hline L4 & 8.91 & 9.20 & 11.86 & 4.92 & 1.61 & 77 \\
\hline L5 & 3.28 & 4.31 & 13.63 & 9.98 & 1.79 & 78 \\
\hline L6 & 8.68 & 30.75 & 9.73 & 9.06 & 3.07 & 62 \\
\hline L7 & 7.63 & 9.29 & 17.83 & 11.37 & 0.51 & 71 \\
\hline L8 & 3.00 & 7.39 & 54.75 & 35.16 & 0.06 & 46 \\
\hline L9 & 6.05 & 7.50 & -3.24 & -3.46 & -8.38 & 102 \\
\hline L10 & 47.63 & 38.14 & 36.80 & 35.01 & 21.74 & 25 \\
\hline L11 & 46.64 & 43.34 & 33.42 & 24.46 & 22.52 & 27 \\
\hline L12 & 26.42 & 19.94 & 13.47 & 13.29 & 10.75 & 53 \\
\hline L13 & 3.35 & 4.89 & 40.02 & 14.72 & -6.53 & 64 \\
\hline L14 & -13.55 & 9.81 & 16.80 & 1.94 & -11.97 & 98. \\
\hline L15 & -1.40 & 17.23 & 25.56 & 10.54 & -19.67 & 92 \\
\hline L16 & -8.69 & -2.40 & 13.41 & -1.28 & -1.36 & 103 \\
\hline L17 & 11.58 & 28.77 & 39.01 & 10.12 & -20.20 & 60 \\
\hline L18 & 0.05 & 0.31 & 8.58 & -0.51 & -8.65 & 101 \\
\hline L19 & 17.95 & 19.80 & 22.80 & 24.53 & 24.06 & 44 \\
\hline L20 & 13.94 & 19.41 & 25.05 & 28.12 & 34.42 & 40 \\
\hline L21 & 27.59 & 28.12 & 29.80 & 25.51 & 24.52 & 36 \\
\hline TMP & 14.71 & 12.11 & 11.76 & 10.60 & 9.44 & 65 \\
\hline
\end{tabular}

higher than that of ligustrazine, may be a potential candidate for intervention in neurological diseases. Furthermore, structure-activity relationship was discussed briefly.

Altogether, results of series of ligustrazinyl amides enrich the study of ligustrazine derivatives with neuroprotective effects. Our completed work supports that the attempt to apply structure combination to discover more efficient neuroprotection lead compounds is viable. Furthermore, to pursue the optimized neuroprotective agents, ligustrazine-cinnamic acid ether and ligustrazinecinnamic acid ester derivatives' neuroprotective effects are ongoing in our lab.

\section{Experimental section Chemistry \\ Materials and methods}

Reactions were monitored by TLC which was performed on silica gel GF254 (Qingdao Haiyang Chemical Co., China) and spots were visualized by modified bismuth potassium iodide or by irradiation with UV light (254 nm). Nuclear magnetic resonance spectra were recorded using a Bruker AVANCE 500 NMR spectrometer (Fällanden, Switzerland) in the indicated solvents. Chemical shifts are expressed in $\delta$ ( $\mathrm{ppm}$ ) relative to tetramethylsilane (TMS). Coupling constants are reported in Hertz $(\mathrm{Hz})$. HRMS spectra were recorded on a Thermo Scientific ${ }^{\text {тм }}$ LTQ Orbitrap XL hybrid FTMS instrument (Thermo Technologies, USA). Melting points (uncorrected) were metered on an X-5 micro melting point apparatus (Beijing, China). Flash column 


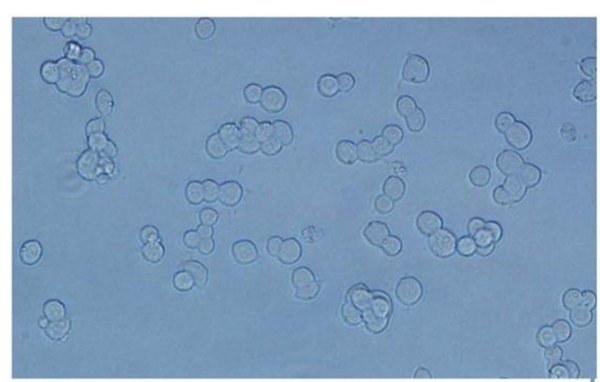

A: Undifferentiated PC12 Cells

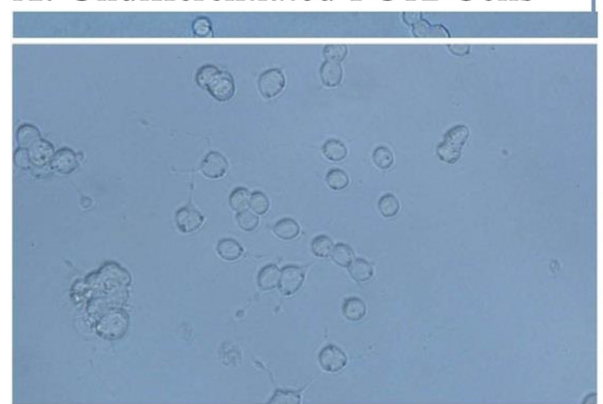

C: $\mathrm{CoCl}_{2}$-insulted PC12 Cells

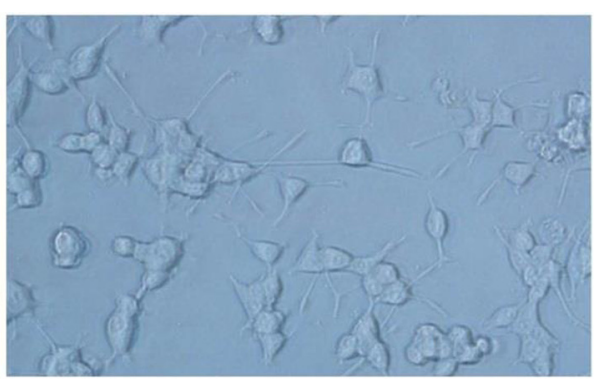

B: Differentiated PC12 Cells

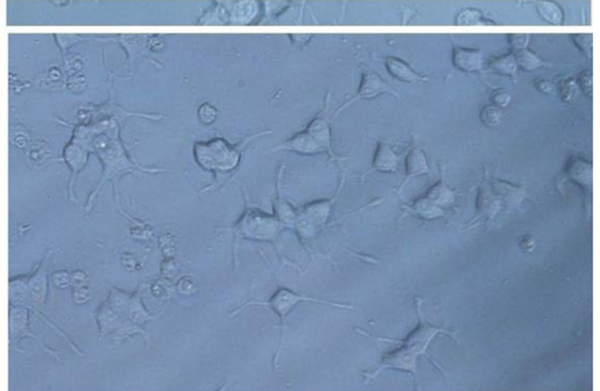

D: $\mathrm{CoCl}_{2}$-insulted PC12 Cells $+\mathbf{L 1 0}$

Figure 1 Effects of L10 on differentiated PC12 cell injury in morphology ( $\times \mathbf{2 0 0})$. (A) Control PC12 cells maintained under normal conditions. (B) PC12 cells exposed to NGF. (C) Differentiated PC12 cells exposed to CoCl 2 insult. (D) Differentiated PC12 cells pre-incubated with L11 then exposed to $\mathrm{CoCl}_{2}$ insult.

chromatography was performed on 200-300 mesh silica gel. All chemicals used were analytical. Solvents were reagent grade or high-performance liquid chromatography grade, and when necessary, were dried by standard methods. Concentration of the reaction solutions involved the use of rotary evaporator at reduced pressure. The yields were calculated by the last step reaction. Among all target compounds, L1, L2, L4, L8, L11, L13, L15, L17, L20 were reported in Liu's research [25]. Therefore, HRMS were supplemented to confirm the chemical structures.

\section{Preparation of (3,5,6-trimethylpyrazin-2-yl)methanamine (L)} The target compound $\mathbf{L}$ was synthesized by three steps. Compound B was prepared according to our previously reported method [27]. The crude product, which caused a strong mucous membrane irritation, could be used in the next step without further purification. To a solution of $\mathbf{B}(5.750 \mathrm{~g}, 27.00 \mathrm{mmol})$ in acetonitrile $(100 \mathrm{~mL})$, phthalimide potassium $(5.000 \mathrm{~g}, 27.00 \mathrm{mmol})$ was added. The mixture was refluxing for $2 \mathrm{~h}$, then concentrated under reduced pressure to afford compound $\mathbf{C}$ (4.878 g, 64\%), white solid. m.p.:155.3-156.8, HRMS (ESI) $\mathrm{m} / \mathrm{z}: 282.12381[\mathrm{M}+\mathrm{H}]^{+}$, calcd. for $\mathrm{C}_{16} \mathrm{H}_{15} \mathrm{~N}_{3} \mathrm{O}_{2}$ 282.12425. The compound $\mathbf{C}$ was purified by flash column chromatography and recrystallization from acetone [28]. The important intermediate $\mathbf{L}$ was obtained by the reaction of $\mathbf{C}(4.000 \mathrm{~g}, 14.23 \mathrm{mmol})$ and $80 \%$ hydrazine hydrate $\left(\mathrm{N}_{2} \mathrm{H}_{4} \cdot \mathrm{H}_{2} \mathrm{O}, 0.85 \mathrm{~mL}\right)$ in absolute ethyl alcohol $(100 \mathrm{~mL})$ refluxing for $5 \mathrm{~h}$. The solution was filtered, concentrated under reduced pressure. The residue was dissolved in dichloromethane, filtered and recovered methylene chloride to give a pale yellow semi-solid substance L (3.521 g, 88\%), HRMS (ESI) m/z: 152.11829 $[\mathrm{M}+\mathrm{H}]^{+}$, calcd. for $\mathrm{C}_{8} \mathrm{H}_{13} \mathrm{~N}_{3}$ 152.12827.

\section{Preparation of L1-L10}

To a solution of $\mathbf{L}(1.324 \mathrm{mmol})$ and the corresponding cinnamic acids $(1.322 \mathrm{mmol})$ in anhydrous $\mathrm{CH}_{2} \mathrm{Cl}_{2}$ $(20 \mathrm{~mL})$, EDCI $(253 \mathrm{mg}, 1.324 \mathrm{mmol})$ and triethylamine $\left(\left(\mathrm{CH}_{3} \mathrm{CH}_{2}\right)_{3} \mathrm{~N}, 3.97 \mathrm{mmol}\right)$ were added. The mixture was stirred at room temperature for $12 \mathrm{~h}$. Then washed with water $(2 \times 20 \mathrm{~mL})$ and brine $(20 \mathrm{~mL})$, successively, dried over sodium sulfate, filtered, and concentrated under vacuum. The residue was purified by flash chromatography and recrystallization from acetone.

$N$-((3,5,6-trimethylpyrazin-2-yl)methyl)cinnamamide (L1): White solid, yield: 90.7\%. HRMS (ESI) $\mathrm{m} / \mathrm{z}$ : 282.15988 $[\mathrm{M}+\mathrm{H}]^{+}$, calcd. for $\mathrm{C}_{17} \mathrm{H}_{19} \mathrm{~N}_{3} \mathrm{O} 282.16064$.

(E)-3-(p-tolyl)-N-((3,5,6-trimethylpyrazin-2-yl)methyl) acrylamide (L2): White solid, yield: 83.5\%. HRMS (ESI) m/ z: 296.17554 $[\mathrm{M}+\mathrm{H}]^{+}$, calcd. for $\mathrm{C}_{18} \mathrm{H}_{21} \mathrm{~N}_{3} \mathrm{O} 296.17629$.

(E)-3-(3,4-dimethoxyphenyl)-N-((3,5,6-trimethylpyrazin-2yl)methyl)acrylamide (L3): White solid, yield: 86.3\%, m.p.: 
150.8-151.4 ${ }^{\circ} \mathrm{C} .{ }^{1} \mathrm{H}$ NMR $\left(500 \mathrm{MHz}, \mathrm{CDCl}_{3}\right): \delta 7.62(\mathrm{~d}, J=$ $15.6 \mathrm{~Hz}, 1 \mathrm{H}), 7.24(\mathrm{~s}, 1 \mathrm{H}), 7.12(\mathrm{~d}, J=8.3 \mathrm{~Hz}, 1 \mathrm{H}), 7.07(\mathrm{~s}$, $1 \mathrm{H}), 6.86(\mathrm{~d}, J=8.3 \mathrm{~Hz}, 1 \mathrm{H}), 6.45(\mathrm{~d}, J=15.6 \mathrm{~Hz}, 1 \mathrm{H}), 4.61$ (d, $J=3.7 \mathrm{~Hz}, 2 \mathrm{H}), 3.92(\mathrm{~s}, 3 \mathrm{H}), 3.91(\mathrm{~s}, 3 \mathrm{H}), 2.53(\mathrm{~s}, 3 \mathrm{H})$, 2.50 (br, $6 \mathrm{H}) ;{ }^{13} \mathrm{C}$ NMR $\left(125 \mathrm{MHz}, \mathrm{CDCl}_{3}\right): \delta 166.2,150.7$, 149.8, 149.2, 147.9, 145.1, 141.0, 128.0, 122.1, 118.6, 111.2, 109.9, 56.1, 56.0, 41.4, 21.5, 21.5, 20.2. HRMS (ESI) m/z: $342.18167[\mathrm{M}+\mathrm{H}]^{+}$, calcd. for $\mathrm{C}_{19} \mathrm{H}_{23} \mathrm{~N}_{3} \mathrm{O}_{3} 342.18177$.

(E)-3-(4-methoxyphenyl)-N-((3,5,6-trimethylpyrazin-2-yl) methyl)acrylamide (L4): White solid, yield: $85.8 \%$. HRMS (ESI) $\mathrm{m} / \mathrm{z}: 312.17093[\mathrm{M}+\mathrm{H}]^{+}$, calcd. for $\mathrm{C}_{18} \mathrm{H}_{21} \mathrm{~N}_{3} \mathrm{O}_{2}$ 312.17120 .

(E)-3-(2-methoxyphenyl)-N-((3,5,6-trimethylpyrazin-2-yl) methyl)acrylamide (L5): White solid, yield: $90.1 \%$, m.p.: 153.3-153.8 ${ }^{\circ} \mathrm{C} .{ }^{1} \mathrm{H}$ NMR $\left(500 \mathrm{MHz}, \mathrm{CDCl}_{3}\right): \delta 7.95(\mathrm{~d}, J=$ $15.8 \mathrm{~Hz}, 1 \mathrm{H}), 7.52(\mathrm{~d}, J=7.5 \mathrm{~Hz}, 1 \mathrm{H}), 7.33-7.30(\mathrm{~m}, 1 \mathrm{H})$, $7.25(\mathrm{~s}, 1 \mathrm{H}), 6.96-6.93(\mathrm{~m}, 1 \mathrm{H}), 6.91(\mathrm{~d}, J=8.3 \mathrm{~Hz}, 1 \mathrm{H})$, $6.67(\mathrm{~d}, J=15.8 \mathrm{~Hz}, 1 \mathrm{H}), 4.61(\mathrm{~d}, J=3.9 \mathrm{~Hz}, 2 \mathrm{H}), 3.88(\mathrm{~s}$, $3 \mathrm{H}), 2.52(\mathrm{~s}, 3 \mathrm{H}), 2.51(\mathrm{~s}, 3 \mathrm{H}), 2.50(\mathrm{~s}, 3 \mathrm{H}) ;{ }^{13} \mathrm{C} \mathrm{NMR}$ $\left(125 \mathrm{MHz}, \mathrm{CDCl}_{3}\right): \delta 166.6,158.3,149.7,148.0,145.2$, $136.5,130.9,128.9,124.0,121.5,120.7,111.2,55.5,41.4$, 21.5, 20.2. HRMS (ESI) $\mathrm{m} / \mathrm{z}: 312.17102[\mathrm{M}+\mathrm{H}]^{+}$, calcd. for $\mathrm{C}_{18} \mathrm{H}_{21} \mathrm{~N}_{3} \mathrm{O}_{2} 312.17120$.

(E)-3-(2,3-dimethoxyphenyl)-N-((3,5,6-trimethylpyrazin2-yl)methyl)acrylamide (L6): White solid, yield: $83.6 \%$, m.p.: $182.7-183.4^{\circ} \mathrm{C} .{ }^{1} \mathrm{H}$ NMR $\left(500 \mathrm{MHz}, \mathrm{CDCl}_{3}\right): \delta 7.94$ $(\mathrm{d}, J=15.9 \mathrm{~Hz}, 1 \mathrm{H}), 7.31(\mathrm{~s}, 1 \mathrm{H}), 7.16(\mathrm{~d}, J=7.8 \mathrm{~Hz}, 1 \mathrm{H})$, $7.06-7.03(\mathrm{~m}, 1 \mathrm{H}), 6.91(\mathrm{~d}, J=8.0 \mathrm{~Hz}, 1 \mathrm{H}), 6.64(\mathrm{~d}, J=$ $15.9 \mathrm{~Hz}, 1 \mathrm{H}), 4.61(\mathrm{~d}, J=2.7 \mathrm{~Hz}, 2 \mathrm{H}), 3.87(\mathrm{~s}, 3 \mathrm{H}), 3.85(\mathrm{~s}$, $3 \mathrm{H}), 2.51$ (s, 3H), $2.50(\mathrm{br}, 6 \mathrm{H}) ;{ }^{13} \mathrm{C}$ NMR $(125 \mathrm{MHz}$, $\left.\mathrm{CDCl}_{3}\right): \delta 166.3,153.3,149.9,148.4,148.1,148.0,145.1$, $135.8,129.3,124.3,122.5,119.5,113.5,61.4,56.0,41.5$, 21.6, 21.6, 20.3. HRMS (ESI) m/z: $342.18155[\mathrm{M}+\mathrm{H}]^{+}$, calcd. for $\mathrm{C}_{19} \mathrm{H}_{23} \mathrm{~N}_{3} \mathrm{O}_{3} 342.18177$.

(E)-3-(3-methoxyphenyl)-N-((3,5,6-trimethylpyrazin-2-yl) methyl)acrylamide (L7): White solid, yield: 91.3\%, m.p.: 117.2-117.9 ${ }^{\circ} \mathrm{C} .{ }^{1} \mathrm{H}$ NMR $\left(500 \mathrm{MHz}, \mathrm{CDCl}_{3}\right): \delta 7.64(\mathrm{~d}, J=$ $15.6 \mathrm{~Hz}, 1 \mathrm{H}), 7.32(\mathrm{~s}, 1 \mathrm{H}), 7.30-7.27(\mathrm{~m}, 1 \mathrm{H}), 7.14$ $(\mathrm{d}, J=7.3 \mathrm{~Hz}, 1 \mathrm{H}), 7.06(\mathrm{~s}, 1 \mathrm{H}), 6.90(\mathrm{~d}, J=7.9 \mathrm{~Hz}$, $1 \mathrm{H}), 6.56(\mathrm{~d}, J=15.6 \mathrm{~Hz}, 1 \mathrm{H}), 4.60(\mathrm{~s}, 2 \mathrm{H}), 3.83(\mathrm{~s}$, 3H), 2.53 (s, 3H), 2.50 (br, 6H); ${ }^{13} \mathrm{C}$ NMR $(125 \mathrm{MHz}$, $\left.\mathrm{CDCl}_{3}\right): \delta 165.9,159.9,149.8,148.0,147.9,144.9$, $141.1,136.3,129.9,121.0,120.6,115.5,113.1,55.4$, 41.4, 21.5, 21.5, 20.2. HRMS (ESI) m/z: $312.17117[\mathrm{M}+\mathrm{H}]^{+}$, calcd. for $\mathrm{C}_{18} \mathrm{H}_{21} \mathrm{~N}_{3} \mathrm{O}_{2} 312.17120$.

(E)-3-(2,5-dimethoxyphenyl)-N-((3,5,6-trimethylpyrazin-2yl)methyl)acrylamide (L8): White solid, yield: $88.5 \%$. HRMS (ESI) $\mathrm{m} / \mathrm{z}: 342.18118[\mathrm{M}+\mathrm{H}]^{+}$, calcd. for $\mathrm{C}_{19} \mathrm{H}_{23} \mathrm{~N}_{3} \mathrm{O}_{3}$ 342.18177 .

(E)-3-(3,4,5-trimethoxyphenyl)-N-((3,5,6-trimethylpyrazin2-yl)methyl)acrylamide (L9): White solid, yield: 81.6\%, m.p.: 154.7-155.4 ${ }^{\circ} \mathrm{C} .{ }^{1} \mathrm{H}$ NMR $\left(500 \mathrm{MHz}, \mathrm{CDCl}_{3}\right): \delta 7.60(\mathrm{~d}, J=$ $15.5 \mathrm{~Hz}, 1 \mathrm{H}), 7.29$ (s, 1H), 6.77 (s, 2H), $6.48(\mathrm{~d}, J=15.5 \mathrm{~Hz}$, $1 \mathrm{H}), 4.61(\mathrm{~s}, 2 \mathrm{H}), 3.89$ (s, 6H), 3.87 (s, 3H), 2.53 (s, 3H),
2.50 (br, $6 \mathrm{H}) ;{ }^{13} \mathrm{C}$ NMR $\left(125 \mathrm{MHz}, \mathrm{CDCl}_{3}\right): \delta 165.9,153.5$, $149.9,148.0,147.9,144.9,141.2,139.5,130.5,120.0,105.1$, 61.1, 56.3, 41.4, 21.6, 21.5, 20.2. HRMS (ESI) m/z: 372.19232 $[\mathrm{M}+\mathrm{H}]^{+}$, calcd. for $\mathrm{C}_{20} \mathrm{H}_{25} \mathrm{~N}_{3} \mathrm{O}_{4} 372.19233$.

(E)-3-(2,3,4-trimethoxyphenyl)-N-((3,5,6-trimethylpyrazin2-yl)methyl)acrylamide (L10): White solid, yield: $85.2 \%, \mathrm{~m}$. p.: $139.8-140.5^{\circ} \mathrm{C} .{ }^{1} \mathrm{H}$ NMR $\left(500 \mathrm{MHz}, \mathrm{CDCl}_{3}\right): \delta 7.81$ (d, $J=15.8 \mathrm{~Hz}, 1 \mathrm{H}), 7.26(\mathrm{~d}, J=8.6 \mathrm{~Hz}, 1 \mathrm{H}), 7.24(\mathrm{~s}, 1 \mathrm{H}), 6.68$ (d, $J=8.6 \mathrm{~Hz}, 1 \mathrm{H}), 6.59$ (d, $J=15.8 \mathrm{~Hz}, 1 \mathrm{H}), 4.61(\mathrm{~d}, J=$ $1.8 \mathrm{~Hz}, 2 \mathrm{H}), 3.91$ (s, 3H), 3.88 (s, 3H), 3.88 (s, 3H), $2.52(\mathrm{~s}$, $3 \mathrm{H}), 2.51$ (br, $6 \mathrm{H}) ;{ }^{13} \mathrm{C}$ NMR (125 MHz, $\left.\mathrm{CDCl}_{3}\right): \delta 166.6$, 155.0, 153.2, 149.8, 148.0, 147.9, 145.2, 142.5, 136.1, 123.4, 122.0, 120.1, 107.6, 61.4, 61.0, 56.1, 41.4, 21.6, 21.5, 20.2. HRMS (ESI) $\mathrm{m} / \mathrm{z}: 372.17950[\mathrm{M}+\mathrm{H}]^{+}$, calcd. for $\mathrm{C}_{20} \mathrm{H}_{25} \mathrm{~N}_{3} \mathrm{O}_{4} 372.19233$.

\section{Preparation of L11-L15, L17-L21}

Intermediate $\mathbf{L}(1.324 \mathrm{mmol})$ and the corresponding phenolic acids $(1.322 \mathrm{mmol})$ were dissolved in anhydrous DMF (L11 $\left.\mathrm{CH}_{2} \mathrm{Cl}_{2}, \mathbf{L 2 1} \mathrm{DMI}\right)(20 \mathrm{~mL}), \mathrm{EDCl}$ (253 mg, $1.324 \mathrm{mmol}$ ) and HOBt $(59 \mathrm{mg}, 0.44 \mathrm{mmol}$ ) were added. The mixture was stirred at room temperature under nitrogen atmosphere for $12 \mathrm{~h}$. The reaction mixture was washed with water $(2 \times 20 \mathrm{~mL})$ and brine $(20 \mathrm{~mL})$, dried over sodium sulfate, filtered, and concentrated under vacuum. The residue was purified by colume chromatography and recrystallization from methanol to give L11-L15 and L17-L21.

2-(((3,5,6-trimethylpyrazin-2-yl)methyl)carbamoyl) phenyl acetate (L11): White solid, yield: 68.9\%. HRMS (ESI) m/z: $336.13163[\mathrm{M}+\mathrm{Na}]^{+}$, calcd. for $\mathrm{C}_{17} \mathrm{H}_{19} \mathrm{~N}_{3} \mathrm{O}_{3}$ 336.13241 .

4-hydroxy-3-methoxy-N-((3,5,6-trimethylpyrazin-2-yl) methyl)benzamide (L12): White solid, yield: 81.8\%, m.p.: 171.3-172.0 $0^{\circ} \mathrm{C} .{ }^{1} \mathrm{H}$ NMR $\left(500 \mathrm{MHz}, \mathrm{CDCl}_{3}\right): \delta 7.84(\mathrm{~s}$, $1 \mathrm{H}), 7.52(\mathrm{~d}, J=1.5 \mathrm{~Hz}, 1 \mathrm{H}), 7.35(\mathrm{dd}, J=8.2,1.5 \mathrm{~Hz}$, $1 \mathrm{H}), 6.95(\mathrm{~d}, J=8.2 \mathrm{~Hz}, 1 \mathrm{H}), 6.40(\mathrm{~s}, 1 \mathrm{H}), 3.93(\mathrm{~s}, 3 \mathrm{H})$, 2.52 (br, 6H), 2.51 (s, 3H); ${ }^{13} \mathrm{C}$ NMR $\left(125 \mathrm{MHz}, \mathrm{CDCl}_{3}\right)$ : $\delta 167.0,149.9,149.0,148.0,148.0,146.8,145.2,126.6$, 120.0, 114.2, 110.6, 56.1, 41.5, 21.6, 21.5, 20.2. HRMS (ESI) $\mathrm{m} / \mathrm{z}: 302.15002[\mathrm{M}+\mathrm{H}]^{+}$, calcd. for $\mathrm{C}_{16} \mathrm{H}_{19} \mathrm{~N}_{3} \mathrm{O}_{3}$ 302.15047 .

4-hydroxy-N-((3,5,6-trimethylpyrazin-2-yl)methyl)benzamide (L13): White solid, yield: 76.9\%. HRMS (ESI) m/z: $272.13925[\mathrm{M}+\mathrm{H}]^{+}$, calcd. for $\mathrm{C}_{15} \mathrm{H}_{17} \mathrm{~N}_{3} \mathrm{O}_{2} 272.13990$.

3-hydroxy-N-((3,5,6-trimethylpyrazin-2-yl)methyl)benzamide (L14): White solid, yield: 78.4\%, m.p.: 210.4$211.3^{\circ} \mathrm{C} .{ }^{1} \mathrm{H}$ NMR (500 MHz, DMSO- $\left.d_{6}\right): \delta 9.64(\mathrm{~s}, 1 \mathrm{H})$, $8.76(\mathrm{t}, J=5.0 \mathrm{~Hz}, 1 \mathrm{H}), 7.31-7.19(\mathrm{~m}, 3 \mathrm{H}), 6.90(\mathrm{dd}, J=$ 7.8, $1.3 \mathrm{~Hz}, 1 \mathrm{H}), 4.51(\mathrm{~d}, J=5.2 \mathrm{~Hz}, 2 \mathrm{H}), 2.46(\mathrm{~s}, 3 \mathrm{H})$, 2.41 (br, $6 \mathrm{H}) ;{ }^{13} \mathrm{C}$ NMR (125 MHz, DMSO- $\left.d_{6}\right): \delta 166.2$, $157.3,149.1,147.9,147.8,147.4,135.7,129.3,118.1$, 117.8, 114.3, 42.1, 21.1, 21.1, 20.3. HRMS (ESI) m/z: $272.13913[\mathrm{M}+\mathrm{H}]^{+}$, calcd. for $\mathrm{C}_{15} \mathrm{H}_{17} \mathrm{~N}_{3} \mathrm{O}_{2} 272.13990$. 
2-hydroxy-N-((3,5,6-trimethylpyrazin-2-yl)methyl)benzamide (L15): White solid, yield: 84.3\%. HRMS (ESI) m/z: 272.13943 $[\mathrm{M}+\mathrm{H}]^{+}$, calcd. for $\mathrm{C}_{15} \mathrm{H}_{17} \mathrm{~N}_{3} \mathrm{O}_{2} 272.13990$.

(E)-3-(4-hydroxyphenyl)-N-((3,5,6-trimethylpyrazin-2-yl) methyl)acrylamide (L17): Little yellow solid, yield: $71.3 \%$. HRMS (ESI) m/z: $296.14035 \quad[\mathrm{M}-\mathrm{H}]^{-}$, calcd. for $\mathrm{C}_{17} \mathrm{H}_{19} \mathrm{~N}_{3} \mathrm{O}_{2} 296.13990$.

(E)-3-(3-hydroxyphenyl)-N-((3,5,6-trimethylpyrazin-2-yl) methyl)acrylamide (L18): Little yellow solid, yield: $74.8 \%$, m.p.:196.3-197.0 $0^{\circ}$. ${ }^{1} \mathrm{H}$ NMR (500 MHz, DMSO- $\left.d_{6}\right): \delta$ $9.60(\mathrm{~s}, 1 \mathrm{H}), 8.52(\mathrm{t}, J=5.0 \mathrm{~Hz}, 1 \mathrm{H}), 7.35$ (d, $J=15.8 \mathrm{~Hz}$, $1 \mathrm{H}), 7.20(\mathrm{t}, J=7.8 \mathrm{~Hz}, 1 \mathrm{H}), 6.97(\mathrm{~d}, J=7.5 \mathrm{~Hz}, 1 \mathrm{H}), 6.93$ $(\mathrm{s}, 1 \mathrm{H}), 6.77(\mathrm{~d}, J=8.0 \mathrm{~Hz}, 1 \mathrm{H}), 6.65(\mathrm{~d}, J=15.8 \mathrm{~Hz}, 1 \mathrm{H})$, $4.47(\mathrm{~d}, J=5.2 \mathrm{~Hz}, 2 \mathrm{H}), 2.46(\mathrm{~s}, 3 \mathrm{H}), 2.43$ (s, 3H), 2.42 (s, $3 \mathrm{H}) ;{ }^{13} \mathrm{C}$ NMR (125 MHz, DMSO- $\left.d_{6}\right): \delta$ 164.8, 157.7, 149.4, 148.0, 147.8, 147.1, 139.2, 136.1, 129.9, 121.7, 118.8, 116.7, 113.7, 41.7, 21.1, 21.0, 20.2. HRMS (ESI) m/z: 296.14037 [M-H] $]^{-}$, calcd. for $\mathrm{C}_{17} \mathrm{H}_{19} \mathrm{~N}_{3} \mathrm{O}_{2} 296.13990$.

(E)-3-(2-hydroxyphenyl)-N-((3,5,6-trimethylpyrazin-2-yl) methyl)acrylamide (L19): Little yellow solid, yield: $77.2 \%$, m.p.: > $210^{\circ} \mathrm{C} .{ }^{1} \mathrm{H}$ NMR $\left(500 \mathrm{MHz}, \mathrm{DMSO}-d_{6}\right): \delta 10.03(\mathrm{~s}$, $1 \mathrm{H}), 8.47(\mathrm{t}, J=5.0 \mathrm{~Hz}, 1 \mathrm{H}), 7.66(\mathrm{~d}, J=15.9 \mathrm{~Hz}, 1 \mathrm{H}), 7.42$ $(\mathrm{d}, J=7.5 \mathrm{~Hz}, 1 \mathrm{H}), 7.17(\mathrm{t}, J=7.6 \mathrm{~Hz}, 1 \mathrm{H}), 6.88(\mathrm{~d}, J=$ $8.1 \mathrm{~Hz}, 1 \mathrm{H}), 6.81(\mathrm{t}, J=7.4 \mathrm{~Hz}, 1 \mathrm{H}), 6.74(\mathrm{~d}, J=15.9 \mathrm{~Hz}$, $1 \mathrm{H}), 4.46(\mathrm{~d}, J=5.2 \mathrm{~Hz}, 2 \mathrm{H}), 2.46(\mathrm{~s}, 3 \mathrm{H}), 2.43$ (s, 3H), 2.42 (s, 3H); ${ }^{13} \mathrm{C}$ NMR (125 MHz, DMSO- $\left.d_{6}\right): \delta 165.4,156.3$, 149.4, 148.0, 147.8, 147.3, 134.8, 130.5, 128.1, 121.6, 121.3, 119.3, 116.1, 41.7, 21.1, 21.0, 20.3. HRMS (ESI) m/z: 296.14047 [M-H] $]^{-}$, calcd. for $\mathrm{C}_{17} \mathrm{H}_{19} \mathrm{~N}_{3} \mathrm{O}_{2} 296.13990$.

(E)-3-(4-hydroxy-3-methoxyphenyl)-N-((3,5,6-trimethylpyrazin-2-yl)methyl)acrylamide (L20): Little yellow solid, yield: $80.1 \%$. HRMS (ESI) m/z: $326.15090[\mathrm{M}-\mathrm{H}]^{-}$, calcd. for $\mathrm{C}_{18} \mathrm{H}_{21} \mathrm{~N}_{3} \mathrm{O}_{3} 326.15047$.

(E)-3-(3,4-dihydroxyphenyl)-N-((3,5,6-trimethylpyrazin-2-yl) methyl)acrylamide (L21): Little yellow solid, yield: 64.3\%, m. p.: $>210^{\circ} \mathrm{C} .{ }^{1} \mathrm{H}$ NMR $\left(500 \mathrm{MHz}, \mathrm{DMSO}-d_{6}\right): \delta 9.43(\mathrm{~s}, 1 \mathrm{H})$, $9.20(\mathrm{~s}, 1 \mathrm{H}), 8.42(\mathrm{t}, J=4.9 \mathrm{~Hz}, 1 \mathrm{H}), 7.26(\mathrm{~d}, J=15.7 \mathrm{~Hz}, 1 \mathrm{H})$, $6.96(\mathrm{~s}, 1 \mathrm{H}), 6.83(\mathrm{~d}, J=7.9 \mathrm{~Hz}, 1 \mathrm{H}), 6.75(\mathrm{~d}, J=8.1 \mathrm{~Hz}, 1 \mathrm{H})$, $6.43(\mathrm{~d}, J=15.7 \mathrm{~Hz}, 1 \mathrm{H}), 4.45$ (d, $J=4.9 \mathrm{~Hz}, 2 \mathrm{H}), 2.45(\mathrm{~s}, 3 \mathrm{H})$, $2.42(\mathrm{~s}, 3 \mathrm{H}), 2.41(\mathrm{~s}, 3 \mathrm{H}) ;{ }^{13} \mathrm{C}$ NMR (125 MHz, DMSO- $\left.d_{6}\right): \delta$ $165.3,149.3,147.9,147.8,147.3,147.3,145.5,139.5,126.3$, $120.4,118.2,115.8,113.9,41.7,21.0,21.0,20.2$. HRMS (ESI) $\mathrm{m} / \mathrm{z}: 336.13140[\mathrm{M}+\mathrm{Na}]^{+}$, calcd. for $\mathrm{C}_{17} \mathrm{H}_{19} \mathrm{~N}_{3} \mathrm{O}_{3}$ 336.13241 .

\section{Preparation of L16}

The desired compound $\mathbf{1 6} \mathbf{b}$ was gained according to the method described by Kojima and Tranchimand with minor modifications [29,30]. EDCI $(0.253 \mathrm{~g}, 1.324 \mathrm{mmol})$ and HOBt $(0.059 \mathrm{~g}, 0.44 \mathrm{mmol})$ were added to a stirred solution of $\mathbf{1 6 b}(0.442 \mathrm{~g}, 1.324 \mathrm{mmol})$ and the intermediate $\mathbf{L}(1.324 \mathrm{mmol})$ in anhydrous $\mathrm{CH}_{2} \mathrm{Cl}_{2}(20 \mathrm{~mL})$. The mixture was stirred at room temperature for $12 \mathrm{~h}$. The mixture was washed with water $(2 \times 20 \mathrm{~mL})$ and brine $(20 \mathrm{~mL})$, dried over sodium sulfate, filtered, and concentrated under vacuum. Flash chromatography afforded 16c (0.566 g, 91.6\%), white solid, m.p.: 127$128^{\circ} \mathrm{C}$, HRMS (ESI) m/z: $490.21033[\mathrm{M}+\mathrm{Na}]^{+}$, calcd. for $\mathrm{C}_{29} \mathrm{H}_{29} \mathrm{~N}_{3} \mathrm{O}_{3}$ 490.21066. To a solution of 16c (0.48 g, $1.028 \mathrm{mmol})$ in methanol $(20 \mathrm{~mL})$, was added $\mathrm{Pd} / \mathrm{C} 10 \%$ $(0.01 \mathrm{~g})$. Then the suspension was stirred under hydrogen atmosphere at room temperature for $12 \mathrm{~h}$. The mixture was filtered, washed with methanol $(2 \times 20 \mathrm{~mL})$, and methanol evaporated under vacuum to afford L16.

3,4-dihydroxy-N-((3,5,6-trimethylpyrazin-2-yl)methyl) benzamide (L16): White solid, yield: $93.7 \%$, m.p.: $>210^{\circ} \mathrm{C}$. ${ }^{1} \mathrm{H}$ NMR (500 MHz, DMSO-d ${ }_{6}$ ): $\delta 9.45(\mathrm{~s}, 1 \mathrm{H}), 9.13$ (s, $1 \mathrm{H}), 8.52(\mathrm{t}, J=5.0 \mathrm{~Hz}, 1 \mathrm{H}), 7.29(\mathrm{~s}, 1 \mathrm{H}), 7.22(\mathrm{~d}, J=$ $8.2 \mathrm{~Hz}, 1 \mathrm{H}), 6.74(\mathrm{~d}, J=8.2 \mathrm{~Hz}, 1 \mathrm{H}), 4.48$ (d, $J=5.0 \mathrm{~Hz}$, 2H), $2.45(\mathrm{~s}, 3 \mathrm{H}), 2.41(\mathrm{~s}, 6 \mathrm{H}) ;{ }^{13} \mathrm{C} \mathrm{NMR}(125 \mathrm{MHz}$, DMSO- $\left.d_{6}\right): \delta 165.9,149.0,148.4,147.8,147.5,144.8$, $125.5,119.0,115.1,114.8,42.1,21.0,21.0,20.2$. HRMS (ESI) $\mathrm{m} / \mathrm{z}: 288.12699[\mathrm{M}+\mathrm{H}]^{+}$, calcd. for $\mathrm{C}_{15} \mathrm{H}_{17} \mathrm{~N}_{3} \mathrm{O}_{3}$ 288.13482 .

\section{Bio-evaluation methods \\ Protective effect on damaged differentiated PC12 cells}

The PC12 cell line (pheochromocytoma) was purchased from Institute of Materia Medica of Chinese Academy of Medical Science. Cells were growed in RPMI 1640 medium supplemented with $5 \%(\mathrm{v} / \mathrm{v})$ fetal bovine serum (FBS), $10 \%(\mathrm{v} / \mathrm{v})$ heat inactivated horse serum and 100 $\mathrm{U} / \mathrm{mL}$ penicillin-streptomycin (Thermo Technologies, USA) and incubated at $37^{\circ} \mathrm{C}$ in a humidified atmosphere of $5 \% \mathrm{CO}_{2}$. When cells achieved the desired density of $>$ $80 \%$ confluency original medium was removed and cells were maintained with serum-free media for $14 \mathrm{~h}$. The cells were resuspended in new growth media which consisted of RPMI 1640 supplemented with 10\% heat inactivated fetal bovine serum. Cells were plated on 96-well dishes pre-coated with poly-L-lysine at $7 \times 10^{3}$ cells/well, differentiated by treated with $50 \mathrm{ng} / \mathrm{mL}$ NGF for $48 \mathrm{~h}$. After these, the differentiated PC12 cells were pretreated with various concentrations $(60,30,15,7.5,3.75 \mu \mathrm{M})$ of ligustrazine derivatives for $36 \mathrm{~h}$. Then the cells were induced by $\mathrm{CoCl}_{2}$ (final concentration, $200 \mathrm{mM}$ ) for $12 \mathrm{~h}$. Control differentiated cells were injected with new growth media at equal amounts. After MTT solution (20 $\mu \mathrm{L}, 5 \mathrm{mg} / \mathrm{mL}$ ) was added to each well, the plate was incubated for a further $4 \mathrm{~h}$ at $37^{\circ} \mathrm{C}$. The supernatant was removed carefully without disturbing the attached cells and formazan crystals were solubilized by adding $100 \mu \mathrm{L}$ DMSO into each well. After shaking for additional $15 \mathrm{~min}$ at $37^{\circ} \mathrm{C}$, the plates were read for optical density at $490 \mathrm{~nm}$ (Thermo Multiskan GO, USA). $\mathrm{CoCl}_{2}$ was dissolved in RPMI 1640 medium. ligustrazine derivatives were dissolved in DMSO [21,31-33]. 
The proliferation rates of damaged $\mathrm{PC} 12$ cells were calculated in the following formula $\left[\mathrm{OD}_{490}\right.$ (Compd) $\left.\mathrm{OD}_{490}\left(\mathrm{CoCl}_{2}\right)\right] /\left[\mathrm{OD}_{490}(\mathrm{NGF})-\mathrm{OD}_{490}\left(\mathrm{CoCl}_{2}\right)\right] \times 100 \%$; the $\mathrm{EC}_{50}$ values were using the equation below: $-\mathrm{pEC}_{50}=$ $\log \mathrm{C}_{\max }-\log 2 \times\left(\Sigma \mathrm{P}-0.75+0.25 \mathrm{P}_{\max }+0.25 \mathrm{P}_{\min }\right)$, Where $\mathrm{C}_{\max }=$ maximum concentration, $\Sigma \mathrm{P}=$ sum of proliferation rates, $\mathrm{P}_{\max }=$ maximum value of proliferation rate and $\mathrm{P}_{\min }=$ minimum value of proliferation rate $[18-21,34]$.

\section{Competing interests}

The authors declare that they have no competing interests.

\section{Authors' contributions}

$\mathrm{HL}$, PW and GL designed the study; GL, XX, KX, FC, JS and SZ performed experiments; GL, BX, YG and $\mathrm{HZ}$ collected and analyzed data; $\mathrm{GL}$ conducted neuroimaging analyses with the help of $Y Z ; G L$ and PW wrote the paper. All authors read and approved the final manuscript.

\section{Acknowledgements}

We gratefully acknowledge support of this work by the National Natura Science Foundation of China (No. 81173519). We also acknowledge the Innovation Team Project Foundation of Beijing University of Chinese Medicine (Lead Compounds Discovering and Developing Innovation Team Project Foundation, No. 2011-CXTD-15)

\section{Author details}

'School of Chinese Pharmacy, Beijing University of Chinese Medicine, No.6 Wangjing Middle Ring South Road, Beijing, Chaoyang District, China. ${ }^{2}$ Department of Pathology, Beijing University of Chinese Medicine, No.11 North Third Ring Road, Beijing, Chaoyang District, China.

Received: 29 July 2014 Accepted: 22 December 2014

Published online: 04 March 2015

\section{References}

1. Lindvall O, Kokaia Z. Stem cells for the treatment of neurological disorders. Nature. 2006;441:1094-6.

2. $Y u$ L, Wang N, Zhang Y, Wang Y, Li J, Wu Q, et al. Neuroprotective effect of muscone on glutamate-induced apoptosis in PC12 cells via antioxidant and $\mathrm{Ca}^{2+}$ antagonism. Neurochem Int. 2014;70:10-21.

3. Feng X, Liang N, Zhu D, Gao Q, Peng L, Dong H, et al. Resveratrol inhibits $\beta$-amyloid-induced neuronal apoptosis through regulation of SIRT1-ROCK1 signaling pathway. PLoS One. 2013;8:e59888.

4. Tereshchenko J, Maddalena A, Bähr M, Kügler S. Pharmacologically controlled, discontinuous GDNF gene therapy restores motor function in a rat model of Parkinson's disease. Neurobiol Dis. 2014;65:35-42.

5. Choi SS, Lee SR, Kim SU, Lee HJ. Alzheimer's disease and stem cell therapy. Exp Neurobiol. 2014;23:45-52.

6. Ginsberg MD. Current status of neuroprotection for cerebral ischemia: synoptic overview. Stroke. 2009;40:S111-4.

7. Xian YF, Lin ZX, Mao QQ, Ip SP, Su ZR, Lai XP. Protective effect of isorhynchophylline against $\beta$-amyloid-induced neurotoxicity in PC12 cells. Cell Mol Neurobiol. 2012;32:353-60.

8. Li JW, Vederas JC. Drug discovery and natural products: end of era or an endless frontier? Science. 2009;325:161-5.

9. Harvey AL. Natural products in drug discovery. Drug Discov Today. 2008;13:894-901

10. Newman DJ, Cragg GM. Natural products as sources of new drugs over the 30 years from 1981 to 2010. J Nat Prod. 2012;75:311-35.

11. Koehn FE, Carter GT. The evolving role of natural products in drug discovery. Nat Rev Drug Discov. 2005;4:206-20.

12. Yu X. Present status and prospect using Ligusticum chuanxiong to prevent and treat stroke. Chin J Integr Med Cardio-/Cerebrovasc Dis. 2006;4:339-41.

13. Kim H. Neuroprotective herbs for stroke therapy in traditional eastern medicine. Neurol Res. 2005:27:287-301.

14. Kao TK, Ou YC, Kuo JS, Chen WY, Liao SL, Wu CW, et al. Neuroprotection by tetramethylpyrazine against ischemic brain injury in rats. Neurochem Int. 2006;48:166-76
15. Zhang C, Wang SZ, Zuo PP, Cui X, Cai J. Protective effect of tetramethylpyrazine on learning and memory function in D-galactose-lesioned mice. Chin Med Sci J. 2004;19:180-4.

16. Lu C, Zhang J, Shi X, Miao S, Bi L, Zhang S, et al. Neuroprotective effects of tetramethylpyrazine against dopaminergic neuron injury in a rat model of Parkinson's disease induced by MPTP. Int J Biol Sci. 2014;10:350-7.

17. Xu K, Wang PL, Xu X, Chu FH, Lin JX, Zhang YZ, et al. An overview on structural modifications of ligustrazine and biological evaluation of its synthetic derivatives. Res Chem Intermediat. 2013. doi:10.1007/s11164-013-1281-2.

18. Cheng XC, Liu XY, Xu WF, Guo XL, Zhang N, Song YN. Ligustrazine derivatives. Part 3: design, synthesis and evaluation of novel acylpiperaziny derivatives as potential cerebrocardiac vascular agents. Bioorg Med Chem. 2009:17:3018-24

19. Li Z, Yu F, Cui L, Zhan P, Wang S, Shen Y, et al. Ligustrazine derivatives. Part 6: design, synthesis and evaluation of novel ligustrazinyl acylguanidine derivatives as potential cardiovascular agents. Med Chem. 2012;8:928-33.

20. Chen H, Li G, Zhan P, Liu X. Ligustrazine derivatives. Part 5: design, synthesis and biological evaluation of novel ligustrazinyloxy-cinnamic acid derivatives as potent cardiovascular agents. Eur J Med Chem. 2011;46:5609-15.

21. Wang P, Zhang H, Chu F, Xu X, Lin J, Chen C, et al. Synthesis and protective effect of new ligustrazine-benzoic acid derivatives against $\mathrm{CoCl}_{2}$-induced neurotoxicity in differentiated PC12 cells. Molecules. 2013;18:13027-42.

22. Picone P, Nuzzo D, Di Carlo M. Ferulic acid: a natural antioxidant against oxidative stress induced by oligomeric A-beta on sea urchin embryo. Biol Bull. 2013;224:18-28.

23. Huang $Y$, Jin M, Pi R, Zhang J, Chen M, Ouyang $Y$, et al. Protective effects of caffeic acid and caffeic acid phenethyl ester against acrolein-induced neurotoxicity in HT22 mouse hippocampal cells. Neurosci Lett. 2013;535:146-51.

24. More SV, Kumar H, Kang SM, Song SY, Lee K, Choi DK. Advances in neuroprotective ingredients of medicinal herbs by using cellular and animal models of Parkinson's disease. Evid Based Complement Alternat Med. 2013;2013:957875.

25. Li Z, Yu F, Cui L, Chen W, Wang S, Zhan P, et al. Ligustrazine derivatives. Part 8: design, synthesis, and preliminary biological evaluation of novel liqustrazinyl amides as cardiovascular agents. Med Chem. 2014;10:81-9.

26. Deng L, Guo X, Zhai L, Song Y, Chen H, Zhan P, et al. Ligustrazine derivatives. Part 4: design, synthesis, and biological evaluation of novel ligustrazine-based stilbene derivatives as potential cardiovascular agents. Chem Biol Drug Des. 2012;79:731-9.

27. Wang P, She G, Yang Y, Li Q, Zhang H, Liu J, et al. Synthesis and biological evaluation of new ligustrazine derivatives as anti-tumor agents. Molecules. 2012;17:4972-85.

28. Al-Qaisi JA, Alhussainy TM, Qinna NA, Matalka KZ, Al-Kaissi EN, Muhi-Eldeen ZA. Synthesis and pharmacological evaluation of a minoacetylenic isoindoline-1,3-dione derivatives as anti-inflammatory agents. Arab J Chem 2011. doi:10.1016/j.arabjc.2010.12.030.

29. Tranchimand S, Tron T, Gaudin C, lacazioa G. First chemical synthesis of three natural depsides involved in flavonol catabolism and related to quercetinase catalysis. Synth Commun. 2006;36:587-97.

30. Kojima T, Hirasa N, Noguchi D, Ishizuka T, Miyazaki S, Shiota Y, et al. Synthesis and characterization of ruthenium(II)-pyridylamine complexes with catechol pendants as metal binding sites. Inorgan Chem. 2010;49:3737-45.

31. Liu Z, Tao X, Zhang C, Lu Y, Wei D. Protective effects of hyperoside (quercetin-3-o-galactoside) to PC12 cells against cytotoxicity induced by hydrogen peroxide and tert-butyl hydroperoxide. Biomed Pharmacother. 2005;59:481-90.

32. Zhou J, Fu Y, Tang XC. Huperzine A and donepezil protect rat pheochromocytoma cells against oxygen-glucose deprivation. Neurosci Lett. 2001:306:53-6.

33. Hu J, Zhao TZ, Chu WH, Luo CX, Tang WH, Yi L, et al. Protective effects of 20-hydroxyecdysone on $\mathrm{CoCl}_{2}$-induced cell injury in PC12 cells. J Cell Biochem. 2010;111:1512-21.

34. Liu X, Zhang R, Xu W, Li C, Zhao Q, Wang X. Synthesis of the novel liqustrazine derivatives and their protective effect on injured vascular endothelial cell damaged by hydrogen peroxide. Bioorg Med Chem Lett. 2003;13:2123-6. 